\title{
Intestinal Obstruction Secondary to Intestinal Intussusception in an Adult, Case Report and Review of the Literature
}

Medina Andrade Luis Angel MD ${ }^{1^{*}}$, Haizel Valencia Romero MD $^{1}$, Lorenzo Hernandez Irving $\mathrm{MD}^{2}$, Zamudio Lizarraga Jennifer $\mathrm{MD}^{2}$, Ordoñez Barrera Ana Silvia $\mathrm{MD}^{2 *}$, Flores Rivera Jesus Christhoper $\mathrm{MD}^{3}$, Alberto Robles Méndez Hernández $\mathrm{MD}^{4}$, Paz Mendoza Adriana $\mathrm{MD}^{4}$, Bastidas Meza Ezequiel Gabriel MD ${ }^{5}$, Sanchez Dauth Dulce Maria MD $^{6}$, Karen Stephanie Reus Muratalla $\mathrm{MD}^{7}$

\author{
${ }^{1}$ General Surgery Service, Hospital General de Zona \#30, IMSS, México. \\ ${ }^{2}$ General Surgery Service, Hospital General de Zona 1A, IMSS, México. \\ *Pathology service \\ ${ }^{3}$ General Surgery Service, Unidad Médica de Alta Especialidad Puebla, IMSS, México. \\ ${ }^{4}$ General Surgery Service, Universidad La Salle, Hospital Angeles Metropolitano, México. \\ ${ }^{5}$ General Surgery Service, Hospital de Especialidades CMNSXXI, México. \\ ${ }^{6}$ General Surgery Service, Hospital Christus Muguerza del Parque, México. \\ ${ }^{7}$ General Surgery Service, Hospital General 1ro de Octubre, ISSSTE
}

*Corresponding Author: Luis Angel Medina Andrade, Hospital General de Zona \#30, Instituto Mexicano del Seguro Social, Mexico City, Mexico,_Email: buismedina_5@hotmail.com

\begin{abstract}
Intussusception of the bowel is defined as the telescoping of a proximal segment of the bowel within the lumen of the distal segment. Intussusception in adults is rare and accounts for only 1-5\% of intestinal obstructions in adults.

Female patients of 45 years old came to hospital complaining for abdominal distention and progressive intolerance to feeding. The last two weeks only drinking water, with multiple throwing episodes. With pathological background of laparoscopic cholecystectomy one month ago. At physical exam with moderate abdominal pain at deep palpation, without clear data of intestinal obstruction, laboratory without alterations. An abdominal CT scan reported a possible intussusception. A laparoscopic exploration confirmed the intussusception that could not be reduced laparoscopically and a transumbilical exteriorization for an intestinal resection was completed without complications. Patient began feeding at fifth day without complications and discharged six days after surgery, with follow up a month after with good evolution. Pathology exam reported a gastrointestinal stromal tumor (GIST).
\end{abstract}

Intussusception in adults is rare but must be considered in the differential diagnosis of intestinal obstruction by the high morbidity associated with the delay in treatment.

Keywords: Adult Intussusception, Intestinal Obstruction, Intestinal Tumor, Acute Abdomen

\section{INTRODUCTION}

Intusuception is the telescoping of a proximal bowel segment into a distal segment, conducing to partial or complete intestinal obstruction. It is more frequent in the first years of life but very rare in the adulthood, with some inespecific simptoms that could difficult the diagnosis.

\section{CASE Report}

Female patients of 45 years old came to hospital complaining for abdominal distention and progressive intolerance to feeding. She refers progressive intolerance the last 6 weeks with worsening the last two weeks and only tolerating water, with multiple throwing episodes. With pathological background of laparoscopic cholecystectomy one month ago and allergies or other pathologies denied. At physical exam she was dehydrated, had moderate abdominal pain at deep palpation specially around umbilicus, without clear data of intestinal obstruction, systemic inflammatory response or laboratory alterations. An 
abdominal CT scan in an external hospital reported a possible intussusception image. A laparoscopic exploration was decided for the increasing pain and confirmed the intussusception that could not be reduced laparoscopically (Fig. 1 and 2)

for this reason a transumbilical exteriorization with resection of intussusception site and $20 \mathrm{~cm}$ of jejunum and anastomosis was completed without complications. Patient began feeding at fifth day without complications and was discharged six days after surgery, with follow up a month after with good evolution. Pathology exam reported a gastrointestinal stromal tumor (GIST)(Fig. 3 and 4).

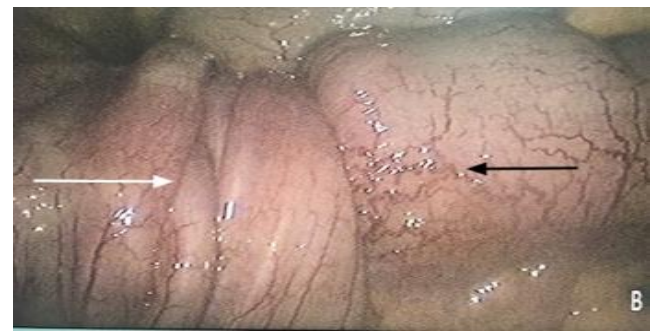

Figure1. Intusuception site, with proximal intestine (black arrow) inside distal segment (White arrow)

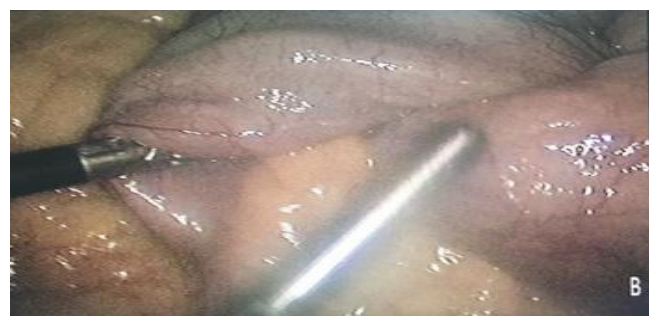

Figure2. Maneouvers for reduction without success

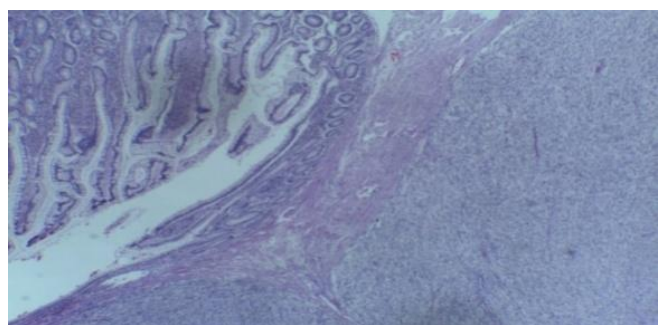

Figure3. Fusocellular mesenchymal neoplasm of submucosal predominance affecting the thickness of the intestinal Wall

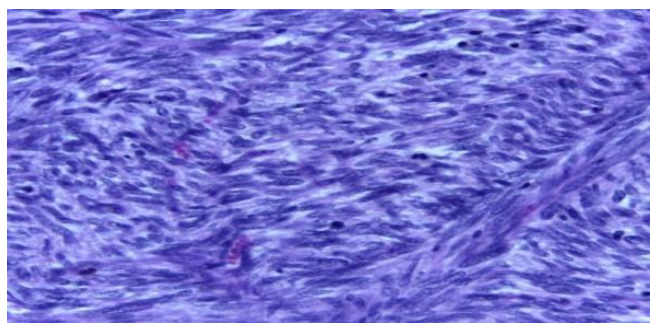

Figure4. Approach at 40x, with intersection of the fusocellular bundles of the neoplasm with apoptic bodies

\section{DISCUSSION}

Intussusception was first described in Amsterdam in 1674 by Barbette, and account for $1-3 \%$ of all cases of intestinal obstruction. It is common in newborns with the classical triad of abdominal pain, palpable abdominal tumor and bloody stool, but these symptoms are not frequent in adults, with other symptoms ranging from constipation, intermittent abdominal pain, partial to complete intestinal obstruction, nausea or vomiting, bleeding per rectum and others, ranging from 1 hour to 4 months since presentation $[1,2]$.

However, the main symptom in different reported studies is abdominal pain (90.9\%), and chronic symptoms in $23.3 \%$. The more frequent presentation in adults is with chronic symptoms of constipation or partial intestinal occlusion, colic and transient pain is also frequent and for this reason the diagnosis could be overlooked. For the previous mentioned reasons, the diagnosis use to be by exclusion of other causes of abdominal pain or intestinal obstruction [3].

Once the surgeon is suspecting the diagnosis the more accurate imaging study is the CT scan, with an accuracy ranging from 58-96.5\%, and the target sign on sagittal view is pathognomonic for the diagnosis $[2,4]$.

In child the main origin is idiopathic, whit only transient intussusception in many cases that could be reversed laparoscopically or manually. In contrast in adults the main origin is demonstrable in $70-90 \%$ of cases, with malignancies in $50-90 \%$ of cases or more depending on the literature, with enteric intussusception being the more common type and, in fact, an study by Jon Wang found that duration of symptoms >14 days, and colonic intussusception were independent predictors for malignancy [2,5].

The optimal treatment is controversial by the high rate of malignancies, some have suggested a laparoscopic approach to treat to reverse intussusception, but many other suggest the laparotomy and resection in block for the previous mentioned facts and need for pathologic study.

The prognosis after surgery is good in general, but it is conditioned by the pathology results when resection is needed, with modification according to the diagnosed tumor type and individual characteristics $[2,4,6]$. 


\section{REFERENCES}

[1] H. Maghrebi, A. Makni, R. Rhaiem, S. Atri, et. al. Adult intussusceptions: Clinical presentation, diagnosis and therapeutic management. International Journal of Surgery Case Reports 33 (2017) 163-166.

[2] Jong Wan Kim , Bong Hwa Lee , Sung Gil Park , Byung Chun Kim, et. al. Factors predicting malignancy in adult intussusception: An experience in university-affiliated hospitals. Asian Journal of Surgery (2018) 41, 92-97.

[3] Kaitlin Kumar, Mahboub R. Noori, Kishan M. Patel, William Yuen, et. al. Rare diagnosis of intestinal lipomatosis complicated by intussusception in an adult: A case report.
International Journal of Surgery Case Reports 39 (2017) 339-342.

[4] Yagan Pilay, An intestinal carcinoid causing transient jejunal intussusception in an adult-A case report. International Journal of Surgery Case Reports 31 (2017) 20-23.

[5] Hannah Samuelson, Dr Giovanni Giannotti, Dr Amy Guralnick. Jejunal lymphangioma causing intussusception in an adult: An unusual case with review of the literatura. Annals of Medicine and Surgery 34 (2018) 39-42.

[6] Siamak Moayedi. Adult intussusception. Visual Journal of Emergency Medicine 9 (2017) 25-26.

Citation: Medina Andrade Luis Angel, Haizel Valencia Romero, Lorenzo Hernandez Irving, Zamudio Lizarraga Jennifer, Ordoñez Barrera Ana Silvia, Flores Rivera Jesus Christhoper, Alberto Robles Méndez Hernández, Paz Mendoza Adriana, Bastidas Meza Ezequiel Gabriel, Sanchez Dauth Dulce Maria, Karen Stephanie Reus Muratalla. Intestinal Obstruction Secondary to Intestinal Intussusception in an Adult, Case Report and Review of the Literature .2019; 5(2):16-18. DOI: http://dx.doi.org/10.2 0431/2455-572X.0502004

Copyright: (C) 2019 Authors. This is an open-access article distributed under the terms of the Creative Commons Attribution License, which permits unrestricted use, distribution, and reproduction in any medium, provided the original author and source are credited. 